\title{
Institutional review board review of clinical research
}

\author{
Beth Taraban, MA, CIP
}

An earlier article in this series ${ }^{1}$ discussed the importance of the development of a sound research question for investigation. Before you actually begin that investigation, however, you will most likely require Institutional Review Board (IRB) approval of your project. IRBs are committees charged with the protection of the participants in your research. While part of the IRB's review will focus on the scientific merit of the proposed research project, the bulk of the review will be directed to issues such as minimizing risks, recruiting subjects equitably, and ensuring that full and voluntary informed consent is obtained from each potential participant prior to beginning any study activity. Traditionally, IRBs have been made up of employees at the institution in which the research is conducted as well as community members and non-scientists. The past decade has seen the rise of "central" or "consortium" IRBs which are not associated with a specific institution but provide reviews-sometimes for a fee-for multiple member institutions. These central IRBs are becoming the review mechanism of choice for multi-site clinical trials, as they can provide a single, efficient mechanism to review a trial taking place at multiple national and international sites.

\section{Historical CONTEXT}

The requirement for committee review to maximize the physical and emotional well-being of the potential participants grew out of a number of violations of the rights and welfare of human subjects that became known during the second half of the $20^{\text {th }}$ century. Chief among these were experiments conducted

Corresponding author: Beth Taraban, MA, CIP Contact Information: beth.taraban@ttuhsc.edu DOI: 10.12746/swrccc 2016.0415.210 on concentration camp prisoners during WWII, the Tuskegee Syphilis Study--a federally funded project that took place from 1932-1972 in which poor African American men, some of whom had syphilis, were never told that they had the disease and were never treated for the condition, even after penicillin became a widely available treatment--and the publication of an influential article $^{2}$ in the New England Journal of Medicine summarizing dozens of ethically questionable research studies. Some of these studies involved infecting children or adults with hepatitis or live cancer cells, withholding penicillin for treatment of strep throat, or taking multiple X-rays of the bladders of healthy infants. Events such as these resulted in the creation of national and international statements of ethical principles regarding the treatment of human subjects in research. Chief among these statements are the 1947 Nuremberg Code ${ }^{3}$, the Declaration of Helsinki $^{4}$, first published in 1964, and the Belmont Report ${ }^{5}$ published by the National Commission for the Protection of Human Subjects of Biomedical and Behavioral Research in 1979. These statements of ethical principles led to the adoption of formal regulations in the US related to the protection of human subjects in research, including the requirement for IRB review and approval of research involving human participants that receives federal funding: Code of Federal Regulations, Title 45, Part 466. These regulations are overseen by the Department of Health and Human Services' Office for Human Research Protections (OHRP). The FDA has similar regulations that apply for research that it oversees. The federal regulations requiring IRB review apply only to research that receives federal funding, but nearly every academic institution in the United States which conducts research with human subjects has indicated in their internal policies that IRB review and approval is required prior to the initiation of ALL research with hu- 
man subjects, regardless of the funding source for the project. A few institutions have adopted less stringent review policies for projects without federal funding, but it is unlikely that any US academic institution would completely waive an ethics review requirement for non-federally funded research.

\section{IRB ReVIEW PROCESS}

Having established, briefly, the historical events that led to the requirement for IRB review of research with human subjects, let us now turn our attention to what a young investigator might expect when a new protocol receives IRB review. Federal regulations require that all IRBs have at least five members. The membership must include at least one person with a scientific background and one member with a non-scientific background. The non-scientist member is the most critical member of the Board, as regulations prohibit any meeting activities from taking place without at least one non-scientist present. Non-scientific IRB members can be recruited from the community (retired teachers, religious leaders, current or former patients) or from within the institution that houses the IRB (administrative assistants, electricians). In addition to at least one scientific and one non-scientific member, all IRBs must have at least one member who is not associated with the institution in any way except by participation on the IRB. This unaffiliated member requirement may have been put in place to decrease the possibility of institutional "groupthink" by the institutional members along the lines of, "Oh, we know Dr. Jones wouldn't submit a bad study. We can just approve this without a thorough review." A majority of the IRB members must be present at a meeting in order for research reviews to take place, and a majority of the members present must agree to approve a project in order for the decision to hold.

\section{IRB APPROVAL REQUIREMENTS}

What does it take for a project to be approved by the IRB? The federal regulations list specific criteria that must be met. IRBs must be able to show that each standard has been adequately addressed before they can vote to approve a study. The regulatory criteria are paraphrased here:

1) Risks to subjects must be minimized by using sound research procedures. Whenever possible, researchers should use procedures that are already being done for purposes of diagnosis or treatment.

2) Risks must be reasonable in relation to anticipated benefits to the subjects themselves or to society at large.

3) Subject selection and recruitment must be equitable with relation to the purposes of the research and must consider whether vulnerable populations are needed.

4) Informed consent will be sought and documented for each participant.

5) When appropriate, there are adequate provisions for monitoring the data being collected.

6) There are adequate provisions to protect the privacy of the subjects and the confidentiality of the data.

7) For subjects who might be vulnerable to coercion or undue influence, there are additional safeguards included.

When the IRB is unable to make all of the determinations required for approval of a research project, its only other options are a) to request changes so that the study can be approved, or b) to disapprove the project. The most common decision upon first review of a project is likely to be a request for modifications in order to secure approval. Though it may be apparent to the study team that they have written a crystal clear protocol, addressing every possible contingency and have provided a straightforward, easily understood consent document, it is often the case that concepts, descriptions, and language that are coherent to the documents' authors are not as clear-cut to a naïve reviewer. The IRB may ask for more details in the Procedures section or for clarification of the information in your recruitment flyer; these changes can lead to stronger, more easily replicated projects, and to better understanding by and protection of the study participants. 


\section{TYPES OF IRB REVIEW}

Not all proposed research involving human subjects has to be reviewed and voted on by a full IRB. Only those projects that are considered to be particularly risky, that involve vulnerable populations, unapproved drugs or devices, or which don't fit neatly into one of the other two categories of human research will require review at a convened meeting of the IRB. These two categories, exempt and expedited projects, can be administratively reviewed (exempt projects) or reviewed and approved by a single qualified IRB member (expedited projects).

Though it would seem reasonable that a project classified as "exempt from IRB review" would not actually require IRB review, a determination of exemption is frequently delegated to an institution's IRB or human research protection office. The federal regulations allow for six categories of research that can be classified as exempt, but the regulators have also recommended that investigators themselves not make their own exemption determinations. For many institutions, then, the exemption decision falls to the IRB. The most common types of projects that receive a determination of exemption include studies that involve 1) the completion of aptitude or achievement tests or innocuous surveys of adults, the results of which are anonymous or would not incur any reasonable risk of harm to the respondents, and 2) reviews of existing data (such as medical records) provided that the data are in existence at the time of the request for exemption and that the recorded data cannot be linked to any identifying information. Details of these and the other four exemption categories can be found in the aforementioned regulations, 45 CFR 46, Section 101.

While studies that can be classified is "exempt" do not require a full approval process, "expedited" studies that meet a set of federally published definitions and which are classified as minimal risk, do require a full review (that is, the project must meet all of the approval criteria presented earlier) by a single qualified IRB member rather than the whole committee. IRB members conducting expedited reviews are only permitted to approve or require modifications in the research in order to secure approval. Any decision to disapprove a research project has to be made at a convened IRB meeting. In academic medical settings, the most common types of projects receiving expedited review are those that involve routine blood draws from healthy adults, noninvasive procedures such as hearing or vision tests, projects requiring moderate exercise and projects involving materials collected only for non-research purposes. While investigators may have a good idea of whether their project will qualify for an expedited review, the authoritative decision will be made by the IRB.

\section{WORKING WITH YOUR IRB}

Armed with an understanding of what an IRB is and how it works, we now address some practical matters related to your IRB submissions, including some strategies for increasing the probability that your project can be reviewed and approved in a timely matter. Perhaps the best piece of advice to be offered is to try to familiarize yourself with the workings of your institution's IRB/human research protection office and the training and submission requirements before preparing any document. While it may be tempting to just ask a colleague for information about how to submit a project for IRB review, you are likely to get more accurate information by seeking out information on your institution's IRB website or by making an appointment to meet with someone in the office. Developing a collegial, mutually respectful relationship with the staff in your human research protection office will be time well spent. The IRB administrators know the common IRB review sticking points, and they will be able to steer you away from them. After all, "clean" IRB submissions are easier for both investigators and IRB members. Another important interpersonal note to remember is that "the IRB" at your institution is not a nameless, faceless group of bureaucrats. Your IRB members are your colleagues and neighbors, who are most likely volunteering their scarce time to serve on what can be a thankless committee. Each member is trying to make the best decision possible about the submission under review given the available information and the regulatory requirements. You will prob- 
ably not always agree with decisions made by your IRB, but it is important to remember that those decisions are not personal, and that the questions you are asked to address or procedures you are asked to change could very well lead to a stronger project. To understand the challenges faced by IRB members at your institution, you might even consider volunteering to serve as an IRB member. You may gain an appreciation of the effort involved in conducting reviews and develop a more tolerant and nuanced perspective of the IRB review process.

As for the actual submission of your project documents for review, the best piece of advice is to proofread all of your documents before submitting them. The importance of careful reading of your documents can't be overstated. The issue isn't with occasional grammatical or typographical errors (these can usually be overlooked by a reviewer unless the documents will be seen by the participants) but with inconsistencies between documents:

"The study protocol indicates that there will be four visits. The informed consent document says that there will be three. Please clarify and correct."

"Inclusion criteria in the application indicate an upper age limit of 45 , but the recruitment flyer indicates an upper age limit of 55 . Please clarify and correct."

"Will participants be completing a quality of life survey? The protocol indicates that they will, but no survey has been provided for review and there is no mention of it in the consent document. Please clarify and correct."

These types of problems are the bane of both IRB reviewers and investigators. They are so simple, but so time-consuming for investigators to address and for IRB members to review (or re-review) before final approval of a study can be given. It's easy to understand how these inconsistencies can happen-an investigator decides partway through preparation of the documents that the $4^{\text {th }}$ study visit won't be necessary, or that the results would be more robust if survey responses were included, but then forgets to change the previously completed documents.
Careful proofreading of all study documents prior to submitting the project to the IRB can help avoid crucial procedural points, since valid research design requires that each participant receives exactly the same intervention. A related piece of advice involves having someone who is unfamiliar with your project review the study documents before you submit them for IRB review. It is easy for authors to forget that all readers are not experts in the proposed area of research and don't have the same knowledge base to understand relationships between variables or your area's common acronyms. An investigator's expertise can lead to pitfalls both in fully explaining the significance of a study's contributions to the field, and in writing consent documents or recruitment materials in a way that can be easily understood by the potential participants. Don't refer to it as a "lower extremity" when you can call it a "leg," for example. We ask our investigators to strive for a $7^{\text {th }}$ grade reading level when preparing study documents that will be seen by participants. For documents that will be reviewed by IRB members, such as your study protocol, consider writing for someone who has no knowledge of your area of research or commonly accepted practices. Also, keep in mind that no IRB will question you for providing too much detail in your description of proposed procedures, but you may very likely be asked to provide additional detail: How will you recruit your volunteers? Who will describe the project? How will you verify that the subject understands the study before s/he signs a consent document? What measures do you have in place in case an adverse event occurs? Where will the data be stored? How will you be sure that private information you collect will be protected? These and multiple other details should be considered, and incorporated into the study documents that the IRB will review.

With a little bit of knowledge and careful planning, your interactions with your Institutional Review Board can be smooth and positive experiences that will soon allow you to start your research project. Stay positive, know that the IRB members do have your research project's success in mind, even as they ask you to make changes in order to secure approval, and best of luck with your future research endeavors. 
Author Affiliations: Beth Taraban is the Assistant Vice President for Research Integrity at Texas Tech University Health Sciences Center.

Submitted: 6/22/2016

Published electronically: 7/15/2016

Conflict of Interest Disclosures: None

Note: Texas Tech University Health Sciences Center's Human Research Protection Program information can be found at http://www.ttuhsc.edu/research/hrpo/irb/

\section{REFERENCES}

1. Peiris A, Lovett C, Tenner T. Your first foray into clinical research. Southwest Respir Crit Care Chron 2016; 4 (14): 17-18.

2. Beecher H. Ethics and clinical research. New Engl J Med 1966; 274 (24): 1354-1360.

3. The Nuremburg Code (1947) In: Mitscherlich, A, Mielke, F. Doctors of infamy: the story of Nazi medical crimes. New York: Schuman, 1949: xxiii-xxv. Access date 22 June, 2016 http:// www.cirp.org/library/ethics/nuremberg/

4. World Medical Association Declaration of Helsinki: Ethical Principles for Medical Research Involving Human Subjects. Revised October, 2000. Access date 22 June, 2016. http://www.fda. gov/ohrms/dockets/dockets/06d0331/06D-0331-EC20-Attach-1. pdf

5. National Commission for the Protection of Human Subjects in Biomedical and Behavioral Research. The Belmont Report: Ethical Principles and Guidelines for the Protection of Human Subjects in Research. April, 1979. Access date 22 June, 2016. http://www.hhs.gov/ohrp/regulations-and-policy/belmont-report/index.html

6. Code of Federal Regulations. Title 45A-Department of Health and Human Services; Part 46-Protection of Human Subjects. Revised, January 15, 2009. Access date 22 June, 2016. http://www.hhs.gov/ohrp/regulations-and-policy/regulations/45-cfr-46/index.html 